\title{
Identification and Antifungal Susceptibility of Candida Isolates from Clinical Samples in a Tertiary Care Teaching Hospital of Central Uttar Pradesh, India
}

\author{
Seema Bose*, Amita Arya and Anjali Agarwal \\ Department of Microbiology, Hind Institute of Medical Sciences, Safedabad, \\ Uttar Pradesh, India \\ *Corresponding author
}

\section{A B S T R A C T}

\begin{tabular}{|c|}
\hline Keywords \\
\hline $\begin{array}{l}\text { Candida albicans, } \\
\text { Non - albicans } \\
\text { Candida, Antifunga } \\
\text { susceptibility, } \\
\text { Ketoconazole }\end{array}$ \\
\hline Article Info \\
\hline $\begin{array}{l}\text { Accepted: } \\
\text { 15 July } 2018 \\
\text { Available Online: } \\
10 \text { August } 2018\end{array}$ \\
\hline
\end{tabular}

\section{Introduction}

Modernization of medical science has caused dramatic changes in patient care. Side by side, it also has introduced emergence and reemergence of certain microorganisms. Due to increasing use of life-saving medical devices, incidence of device-associated infection is rising steadily. Previously, fungal infection was usually restricted to cutaneous and mucocutaneous surfaces. Now it has emerged as life- threatening pathogenic organism all over the world. Candidiasis is also a major cause of morbidity and mortality in terminally
Candida albicans (C.albicans) is considered as the commonest cause of Candidiasis. But recently incidence of non - albicans Candida (NAC) infection has increased. The most commonly used drugs against candidiasis are azole derivatives. Resistance pattern of different Candida species (spp) varies widely, some of them being inherently resistant. This study was undertaken to find out incidence of Candidiasis, their species identification and susceptibility of azole derivatives. In 6 months period, total 43 Candida spp. were isolated from various clinical samples, among which $C$. albicans $(46.5 \%), C$. tropicalis $(24.5 \%), C$. glabrata (23.4\%) and C. krusie (4.6\%).Susceptibility to Ketoconazole (KT), Fluconazole (FLC) and Itraconazole (IT) were detected. 
This study was undertaken to identify Candida isolates from various clinical samples up to species level and to find out their antifungal susceptibility.

\section{Material and Methods}

This was a prospective, cross - sectional study. The study period is 6 months. Clinical samples like blood, urine, pus, sputum, catheter tips, vaginal swabs and throat swabs were processed in the microbiology department. In this study, all Candida isolates from culture of various clinical specimens were further tested for species identification and antifungal susceptibility.

Inclusion criteria - Candida isolates from all clinical specimen in pure culture.

Exclusion criteria - Repeat isolates from same clinical specimen of same patient and isolation of Candida species from mix culture.

Candida spp. is a commensal of vagina and buccal cavity. Therefore, demonstration on gram staining of smear and isolation in culture were done to diagnose cases of oropharyngeal and vulvovaginal candidiasis. In urine sample, colony counts of $>10^{5} \mathrm{cfu} / \mathrm{ml}$ was considered as significant candiduria in patients without indwelling catheters. In catheterized patients, $1000 \mathrm{cfu} / \mathrm{ml}$ indicated significant candiduria. Isolation of very less no of Candida spp was considered significant from sterile body fluids like blood, cerebrospinal fluid, pleural fluid and peritoneal fluid (Kauffman CA.2005).

All culture isolates were identified up to species level following standard mycological protocol, such as; germ tube production, chlamydospore formation, carbohydrate assimilation, colony morphology and pigment production on Hi-Chrom Candida agar (Hi- media Laboratories Pvt Ltd, India) (Deorukhkar, 2014.). Hi-Candida identification kit (Hi-media Laboratories Pvt. Ltd, India) was also used to identify Candida isolates. For this, test was done as per instruction of manufacturer.

Antifungal sensitivity of Candida isolates was done by Kirby-Bauer disc diffusion method. Mueller Hinton agar supplemented with $0.2 \%$ glucose and $0.5 \mu \mathrm{g} / \mathrm{ml}$ methylene blue dye medium (MH-GMB) was used for this purpose (Clinical and Laboratory Standards Institute [CLSI]. 2004.). Azole group of Antifungal drug discs, such as; Fluconazole $(25 \mu \mathrm{g})$, Ketoconazole $(10 \mu \mathrm{g})$, Itraconazole $\left(\begin{array}{ll}10 & \mu \mathrm{g}\end{array}\right)$ were procured from Hi-media Laboratories Pvt Ltd India.

For preparation of inoculum, $24 \mathrm{~h}$ old culture grown on Sabouraud's dextrose agar (SDA) was used. For sensitivity test, 4-5 distinct colonies were suspended in $5 \mathrm{ml}$ of sterile $0.85 \%$ saline and then; inoculum was spread on entire surface of MH-GMB with help of sterile cotton swab. Azole discs were placed on to the surface of media by using sterile forceps. Inoculated plates were incubated at $37 \mathrm{oC}$ for $24 \mathrm{~h}$. Antifungal susceptibility testing were interpreted as per the approved Clinical and Laboratory Standard Institute guidelines (CLSI. 2004, CLSI,2002,Pfaller M. A.et al., 2012).

Itraconazole and Fluconazole resistance was confirmed by quantitative determination of susceptibility of fungus to antifungal agent using MIC strip, obtained from $\mathrm{Hi}$ - media Pvt Ltd, India. The test was performed as per manufacturer's instruction.

C. albicans (ATCC 90028), C. parapsilosis (ATCC 22019), C. tropicalis (ATCC 750) and C. krusei (ATCC 6258) were used as reference strains for quality control (Pfaller $e t$ al., 2012). 


\section{Results and Discussion}

From 1003 total clinical samples, we isolated 43 Candida spp among which, there were 20 C. albicans $(46.5 \%), 11$ C. tropicalis $(24.5 \%)$, 10 C. glabrata $(23.4 \%)$ and 2 C. krusei (4.6\%) (Table1). C. krusie showed 100\% resistance to KT and FLC and 50\% resistance to IT. C. glabrata showed only $10 \%$ resistance to KT and all glabrata spp were susceptible to FLC and IT. C. albicans isolates were $25 \%$ resistant to $\mathrm{KT}, 15 \%$ to FLC and $10 \%$ to IT where as, C. tropicalis showed $27.3 \%$ resistance to KT and FLC both and $15 \%$ resistance to IT.

In recent years, incidence of fungal infection has significantly increased. Several factors, such as; emergence of HIV/AIDS, widespread use of immunosuppressive drugs, indiscriminate use of broad spectrum antibiotics, introduction of many life saving medical devices, biofilm formation and invasive surgical interventions might be the underlying predisposing factors (Kobayashi et al., 2004).

In the present study, maximum number of candida isolates was $C$. albicans, followed by C. tropicalis, C. glabrata and C. krusie. This finding is similar to the work done by some other researchers (Kobayashi et al., 2004, lvarez-Lerma et al., 2003, Gonzalez Gravina et al., 2007). Predisposing factors for emergence of NAC group as pathogen may be due to prematurity, use of broad-spectrum antibiotics, immunosuppression and empirical use of antimycotic drugs. Clinical manifestations of infections by different NAC spp may be same but resistance pattern to antifungal drugs are different because some of them are inherently resistant or acquire resistance, or both, to antimycotic drugs (Sullivan et al., 1996). In our study, antifungal susceptibility of Candida isolates was done by disc diffusion technique. Disc diffusion method for yeast is similar to the routinely used Kirby-Bauer method done for antibiotic susceptibility testing (Rex et al., 2001). It is relatively cost effective and convenient method, which can be readily done in routine laboratories (Deorukhkar et al., 2017).

We also detected MIC of Itraconazole and Fluconazole. Although qualitative results obtained by disk diffusion method are useful in routine laboratory diagnosis, quantitative MIC detection is sometimes required for treatment of invasive infection. Other researchers also observed that e-strip test is quite simple, rapid, cost effective and can be easily done in routine laboratory practice. They also reported that this method could produce similar results as in reference methods for yeast (Ana et al., 2015).

Fluconazole resistance was significantly high among NAC spp like C. krusei (100\%), C. glabrata (2\%) and C. tropicalis (21\%). C. albicans was $30.1 \%$ resistant to fluconazole. C. tropicalis, which initially considered as fluconazole sensitive; now a days has been reported as highly resistant as reported in various studies (Pfaller, 2007; Yang, 2004). Reason for this is not known yet and this can be a future research topic for investigators. Equally, significantly high incidence of resistance against other azole drugs was observed in this study. C. glabrata was found to be least resistant among all the Candida isolates (Table 1).

Azole group of antifungal agents is most commonly used Antifungal agent for the treatment of candidiasis. These drugs are safe with less side effects and act against all clinical types of Candidiasis. Therefore, resistance to these drugs is a matter of concern (Me' an et al., 2008). Mechanisms of such resistance can be due to modifications of target enzymes, low access of the drug to the target, or a combination of both (Silva et al., 2012). 
Table.1 Distribution of different Candida isolates $(\mathrm{n}=43)$

\begin{tabular}{|l|l|}
\hline Fungal isolates & Number (\%) \\
\hline C.albicans & $20(46.5 \%)$ \\
\hline C.tropicalis & $11(24.5 \%)$ \\
\hline C.glabrata & $10(23.4 \%)$ \\
\hline C. krusie & $02(4.6 \%)$ \\
\hline
\end{tabular}

Table 1 the highest number of isolate was C. albicans, followed by C. tropicalis, C. glabrata and C. krusei.

Table.2 Antifungal resistance pattern of Candida isolates $n=43$ )

\begin{tabular}{|l|l|l|l|}
\hline Candida isolates & $\begin{array}{l}\mathrm{KT}(10 \mu \mathrm{g}) \text { No of } \\
\text { resistant strain }(\%)\end{array}$ & $\begin{array}{l}\text { FLC }(25 \mu \mathrm{g}) \text { No of } \\
\text { resistant strain }(\%)\end{array}$ & $\begin{array}{l}\text { IT }((10 \mu \mathrm{g}) \text { No of } \\
\text { resistant strain (\%) }\end{array}$ \\
\hline C.albicansn=20 & $5(25 \%)$ & $3(15 \%)$ & $2(10 \%)$ \\
\hline C. tropicalisn= 11 & $3(27.3 \%)$ & $3(27.3 \%)$ & $2(15 \%)$ \\
\hline C.glabratan=10 & $1(10 \%)$ & $00(00)$ & $00(00)$ \\
\hline C.krusien= 02 & $2(100 \%)$ & $2(100 \%)$ & $1(50 \%)$ \\
\hline
\end{tabular}

Table 2 Resistance patterns of Candida isolates to azole derivatives. C. krusie showed 100\% resistance to KT and FLC and 50\% resistance to IT. C. glabrata showed only $10 \%$ resistance to KT and all glabrata spp were susceptible to FLC and IT.

In conclusion, this study identified Candida spp. isolated from various clinical specimens and their susceptibility pattern to azole derivatives from this part of India. Increasing resistance pattern of Candida spp to azole derivatives was noted. Species identification and antifungal susceptibility testing of Candida spp. should be done regularly to help the treatment process of mycotic infection.

\section{Acknowledgments}

Authors are grateful to the management of Hind Institute of Medical Sciences, Barabanki, UttarPradesh, India, for their encouragement and support throughout the study.

\section{References}

Ana A-I, Marcia SCM, Rodriguez T.2015. Susceptibility test for fungi: clinical and laboratorial correlations, Med Mycol; 57 (suppl 19): $57-64$.

Clinical and Laboratory Standards Institute
(CLSI),2002. Reference Method For Broth Dilution Antifungal Susceptibility Testing of Yeasts, Approved standard M27-A2, Clinical Laboratory Standard Institute, Wayne, Ind, USA, 2nd edition.

Clinical and Laboratory Standards Institute (CLSI). 2004. Reference Method For Antifungal Disk diffusion Susceptibility Testing of Yeasts, Approved standard M44-A, Clinical Laboratory Standard Institute, Wayne, Ind, USA, 2nd edition.

Deorukhkar S C, Roushani S. 2017. Fluconazole resistance in Candida Species: Ten Year's Experience at a Rural Tertiary Care Teaching Hospital in India. J Infect Dis Pathol 1: 102.

Deorukhkar S. C. and Saini S. 2014. Laboratory approach for diagnosis of candidiasis through ages, Int $\mathbf{J}$ Current Microbiol and Appl Sci, 3, 1, 206-218.

Gonzalez Gravina H, Gonzalez de Moran E, Zambrano O, Lozano Chourio $\mathrm{M}$, Roudriquez de Valero S, et al., 2007. Oral candidiasis in children and adolescents with cancer. Identification of Candida spp. Med Oral Pathol Oral Cir Bucal 12: 
E419-23.

Kauffman CA. 2005. Candiduria. Clin Infect Dis; 41(suppl 6): $5371-6$.

Kobayashi CC, de Fernandes OF, Miranda KC, de Sousa ED, Silva Mdo R. 2004. Candiduria in hospital patients: a study prospective. Mycopathologia 158: 49-52.

lvarez-Lerma F. A', Nolla-Salas J., Leo' n C. et al., 2003. Candiduria in critically ill patients admitted to intensive care medical units. Intensive Care Med, 29, 7, 1069-1076.

M. A. Pfaller and D. J. Diekema. 2007. Epidemiology of invasive candidiasis: a persistent public health problem. Clin Microbiol Rev, 20, 1, 133-163.

M. Me' an, O. Marchetti, and T. Calandra. 2008. Bench-to-bedside review: Candida infections in the intensive care unit. Critical Care, 12, 1, article 204.

Pfaller M. A. and Diekema D. J. 2012. Progress in antifungal susceptibility testing of Candida spp. by use of Clinical and Laboratory Standards Institute broth microdilution methods, 2010 to 2012 , J Clin Microbiol, 50, 9, 2846-2856.

Rex JH, Pfaller M, Walsh T, Chaturvedi V, Espinel-Ingro A, et al., 2001. Antifungal susceptibility testing: Practical aspects and current challenges. Clin Microbiol
Rev 14: 643-58.

S. Silva, M. Negri, M. Henriques, R. Oliveira, D. W. Williams, and J. Azeredo. 2012. Candida glabrata, Candida parapsilosis and Candida tropicalis: biology, epidemiology, pathogenicity and antifungal resistance. FEMS Microbiol Rev, 36, 288-305.

Sardi J. C. O., Scorzoni L., Bernardi T., FuscoAlmeida A. M., and Mendes Giannini M. J. S. 2013. Candida species: current epidemiology, pathogenicity, bio film formation, natural antifungal products and new therapeutic options, J Med Microbiol, 62, 1, 10-24.

Snydman D. R. 2003. Shifting patterns in the epidemiology of nosocomial Candida infections, Chest, 123, 5, 500S- 503S.

Sullivan D. J., Henman M. C., Moran G. P. et al., 1996.Molecular genetic approaches to identification, epidemiology and taxonomy of non-albicans Candida species, J Med Microbiol, 44, 6, 399-408.

Y-L Yang, Ho, H. H Cheng, M. Ho, and H J Lo. 2004. Susceptibilities of Candida species to amphotericin B and fluconazole: the emergence of fluconazole resistance in Candida tropicalis. Infection control and hospital epidemiology, 25,1, 60-64

\section{How to cite this article:}

Seema Bose, Amita Arya and Anjali Agarwal. 2018. Identification and Antifungal Susceptibility of Candida Isolates from Clinical Samples in a Tertiary Care Teaching Hospital of Central Uttar Pradesh, India. Int.J.Curr.Microbiol.App.Sci. 7(08): 2665-2669.

doi: https://doi.org/10.20546/ijcmas.2018.708.276 